Pacific Journal of Mathematics

THE SCHUR SUBGROUP OF THE BRAVER GROUP 


\section{THE SCHUR SUBGROUP OF THE BRAUER GROUP}

\section{J. William Pendergrass}

Let $K$ be a subfield of a cyclotomic extension $L$ of the rational field $Q$. The Schur subgroup, $S(K)$, of the Brauer group of $K, B(K)$, consists of those algebra classes which contain an algebra which is isomorphic to a simple component of a group algebra $Q G$ for some finite group $G$.

In this paper we describe a set of generators for $S(K)$. We then use this theorem to determine the 2-primary part of $S(K)$ when $L / K$ is cyclic and the fourth roots of unity are not in $K$.

Notation. In this paper $K$ is a field contained in $Q\left(\varepsilon_{n}\right)$ where $\varepsilon_{n}$ is a primitive $n$th root of unity. The completion of $K$ at a prime $P$ is denoted $K_{P}$. If $p$ is the integral prime dividing $P$, then the residue class degree of $P$ over $p$ is written $f(p)=f(p, K / Q)$. The ramification index of $p$ in $Q\left(\varepsilon_{n}\right)$ over $K$ is $e(p)=e\left(p, Q\left(\varepsilon_{n}\right) / K\right)$.

If $A$ is a central simple algebra over $K$, then [A] will denote the class of $A$ in $B(K)$. A class [A] in $B(K)$ is said to have uniformly distributed invariants of values 0 or $1 / 2$ if for each rational prime $p$, $[A]$ has the same Hasse invariant at each of the primes of $K$ which divide $p$, and these invariants are either 0 or $1 / 2$. The common value of the invariant of $[A]$ at the primes of $K$ dividing $p$ is called the $p$-local invariant of $[A]$ and is denoted: $\operatorname{inv}_{p}[A]$.

If $L$ is an extension field of $K$, then the Galois group of $L$ over $K$ is denoted by $\mathrm{Gal}(L / K)$, and the Frobenius automorphism of a prime $p$ unramified in $L$ over $K$ is written $[L / K, p]$. Let $\alpha$ be a factor set $\operatorname{Gal}(L / K) \times \operatorname{Gal}(L / K)$ into $L$. Then the crossed product algebra made with $L$ and $\alpha$ is denoted by $(L / K, \alpha)$. This is a central simple $K$ algebra having $L$ basis $\left\{u_{\sigma}\right\}$ for $\sigma \in \operatorname{Gal}(L / K)$ with multiplication given by

$$
\begin{aligned}
u_{\sigma} u_{\tau} & =\alpha(\sigma, \tau) u_{\sigma \tau} \\
u_{\sigma} x & =\sigma(x) u_{\sigma} \quad \text { for } \quad \sigma, \tau \in \operatorname{Gal}(L / K), \quad x \in L .
\end{aligned}
$$

In case $\operatorname{Gal}(L / K)=\langle\sigma\rangle$ is cyclic, we shall write $(L, \sigma, a)$ for the crossed product in which

$$
\begin{aligned}
\left(u_{\sigma}\right)^{i} & =u_{\sigma^{i}} & & 1 \leqq i<|\sigma| \\
& =a & & i=|\sigma| .
\end{aligned}
$$

If $p$ is a rational prime which splits into an even number of primes in $K$ over $Q$, then $\Omega(p)$ denotes the class of $B(K)$ with invariant $1 / 2$ at each of the primes of $K$ dividing $p$ and invariant 
0 elsewhere. If $p_{1}$ and $p_{2}$ are rational primes which split into an odd number of primes in $K$ over $Q$, then $\Omega\left(p_{1}, p_{2}\right)$ denotes the class in $B(K)$ with invariant $1 / 2$ at each of the primes of $K$ dividing $p_{1} p_{2}$ and invariant 0 elsewhere.

Finally $|m|_{2}$ denotes the highest power of 2 which divides the integer $m$, and $t(q)=q^{f(q)}-1$ for all rational primes $q$.

2. The generator theorem. In this section we give a set of generators for $S(K)$. This is a useful refinement of a result by Janusz [6].

LEMMA 1. Let $K$ be a field contained in $Q\left(\varepsilon_{n}\right)$ where $n$ is odd. Suppose that Gal $\left(Q\left(\varepsilon_{n}\right) / K\right)=\prod_{i=1}^{r}\left\langle\phi_{i}\right\rangle$ and that $\operatorname{Gal}\left(Q\left(\varepsilon_{4 n}\right) / Q\left(\varepsilon_{n}\right)\right)=\langle\rho\rangle$. If $\left[Q\left(\varepsilon_{n}\right) / K, 2\right]=\Pi \phi_{i}^{g_{i}}$, then the 2-local index of an algebra $\left(Q\left(\varepsilon_{4 n}\right) / K, \alpha\right)$ is equal to 2 if and only if $\sum g_{i} x_{i}+z f(2)$ is odd where $u_{\rho} u_{\phi_{i}}=\varepsilon_{4}^{x_{i}} u_{\phi_{i}} u_{\rho}$ and $u_{\rho}^{2}=\varepsilon_{4}^{2 z}$.

Proof. Set $\eta=\left[Q\left(\varepsilon_{n}\right) / K, 2\right]$ and suppose that $\eta$ has order $s$. Then $u_{\eta} u_{\rho}=\varepsilon_{4}^{\lambda} u_{\rho} u_{\eta}$ where

$$
\lambda=\sum_{i=1}^{r} g_{i} x_{i}
$$

If $\lambda$ is even we have

$$
u_{\rho}\left(\varepsilon_{4}^{\lambda / 2} u_{\eta}\right)=\varepsilon_{4}^{-\lambda / 2} \varepsilon_{4}^{\lambda} u_{\eta} u_{\rho}=\left(\varepsilon_{4}^{\lambda / 2} u_{\eta}\right) u_{\rho} .
$$

Let $\pi$ be a prime of $K$ dividing 2 , then

$$
\begin{aligned}
K_{\pi \otimes K}\left(Q\left(\varepsilon_{4 \eta}\right) / K, \alpha\right) & =\sum_{i=0}^{1} \sum_{j=0}^{s-1} Q_{2}\left(\varepsilon_{4 \eta}\right) u_{\rho}^{i} u_{\eta}^{j} \\
& =\sum_{i=0}^{1} \sum_{j=0}^{s-1} K_{\pi}\left(\varepsilon_{4}\right) Q_{2}\left(\varepsilon_{\eta}\right) u_{\rho}^{i}\left(\varepsilon_{4}^{\lambda / 2} u_{\eta}\right)^{j} \\
& =\left(\sum_{i=0}^{1} K_{\pi}\left(\varepsilon_{4}\right) u_{\rho}^{i}\right)\left(\sum_{j=0}^{s-1} Q_{2}\left(\varepsilon_{n}\right)\left(\varepsilon_{4}^{\lambda / 2} u_{\eta}\right)^{j}\right) \\
& =\left(K_{\pi}\left(\varepsilon_{\eta}\right), \rho, u_{\rho}^{2}\right) \bigotimes_{K_{\pi}}\left(Q_{2}\left(\varepsilon_{\eta}\right), \eta,\left(\varepsilon_{4}^{\lambda / 2} u_{\eta}\right)^{s}\right) .
\end{aligned}
$$

Now $\left(\varepsilon_{4}^{\lambda / 2} u_{\eta}\right)^{8}$ is a root of unity and $Q_{2}\left(\varepsilon_{n}\right)$ is unramified over $K_{\pi}$, hence by [1, Chap. V, Thm. 9.14] $\left(Q_{2}\left(\varepsilon_{n}\right), \eta,\left(\varepsilon_{4}^{\lambda / 2} u_{\eta}\right)^{8}\right)$ has index 1 . Further

$$
\left[\left(K_{\pi}\left(\varepsilon_{4}\right), \rho, \varepsilon_{4}^{2 z}\right)\right]=\left[K_{\pi} \bigotimes_{Q_{2}}\left(Q_{2}\left(\varepsilon_{4}\right), \rho, \varepsilon_{4}^{2 z}\right)\right]
$$

and $\left(Q_{2}\left(\varepsilon_{4}\right), \rho, \varepsilon_{4}^{2 z}\right)$ has index 2 if and only if $z$ is odd, since -1 is not a norm from $Q_{2}\left(\varepsilon_{4}\right)$. Thus $K_{\pi} \otimes_{K}\left(Q\left(\varepsilon_{4 n}\right) / K, \alpha\right)$ has index 2 if and only if $f(2) z$ is odd in the case that $\lambda$ is even.

Now suppose that $\lambda$ is odd. We have that 


$$
u_{\rho}\left(\left(1+\varepsilon_{4}^{\lambda}\right) u_{\eta}\right)=\left(1+\varepsilon_{4}^{-\lambda}\right) \varepsilon_{4}^{\lambda} u_{\eta} u_{\rho}=\left(\left(1+\varepsilon_{4}^{\lambda}\right) u_{\eta}\right) u_{\rho} .
$$

Hence

$$
\left[K_{\pi} \bigotimes_{K}\left(Q\left(\varepsilon_{4 n}\right) / K, \alpha\right)\right]=\left[\left(K_{\pi}\left(\varepsilon_{4}\right), \rho, u_{\rho}^{2}\right) \bigotimes_{K_{\pi}}\left(Q\left(\varepsilon_{n}\right), \eta,\left(\left(1+\varepsilon_{4}^{\lambda}\right) u_{\eta}\right)^{s}\right)\right]
$$

by the same reasoning used above. We have already seen that $\left(K_{\pi}\left(\varepsilon_{4}\right), \rho, u_{\rho}^{2}\right)$ has index 2 if and only if $f(2) z$ is odd; we must look at $\left(Q_{2}\left(\varepsilon_{n}\right), \eta,\left(\left(1+\varepsilon_{4}^{\lambda}\right) u_{\eta}\right)^{s}\right)$.

Let $V_{L}$ denote the exponential valuation in the 2 -adic field $L$. Then

$$
\begin{aligned}
V_{K_{\pi}}\left(\left(1+\varepsilon_{4}^{\lambda}\right) u_{\eta}\right)^{s} & =\frac{1}{2} V_{K_{\pi}\left(\varepsilon_{4}\right)}\left(\left(1+\varepsilon_{4}^{\lambda}\right) u_{\eta}\right)^{s} \\
& =\frac{1}{2} V_{K_{\pi}\left(\varepsilon_{4}\right)}\left(1+\varepsilon_{4}^{\lambda}\right)^{s}+\frac{1}{2} V_{K_{\pi}\left(\varepsilon_{4}\right)}\left(u_{\eta}^{s}\right) \\
& =\frac{s}{2} V_{K_{\pi}\left(\varepsilon_{4}\right)}\left(1+\varepsilon_{4}^{\lambda}\right)
\end{aligned}
$$

since $u_{\eta}^{s}$ is a unit in $K_{\pi}\left(\varepsilon_{4}\right)$. Further, $\left(1+\varepsilon_{4}^{\lambda}\right)$ is a prime element in $K_{\pi}\left(\varepsilon_{4}\right)$ since $\lambda$ is odd. Thus $V_{K_{\pi}\left(\varepsilon_{4}\right)}\left(1+\varepsilon_{4}^{\lambda}\right)=1$ and

$$
V_{K_{\pi}}\left(\left(1+\varepsilon_{4}^{\lambda}\right) u_{\eta}\right)^{s}=s / 2 .
$$

Hence, by the definition of the Hasse invariant,

$$
\begin{aligned}
\operatorname{inv}\left(Q_{2}\left(\varepsilon_{n}\right), \eta,\left(\left(1+\varepsilon_{4}^{\lambda}\right) u_{\eta}\right)^{s}\right) & =\frac{s / 2}{s} \bmod Z \\
& =\frac{1}{2} \bmod Z .
\end{aligned}
$$

Therefore, if $\lambda$ is odd, we have that the index of $K_{\pi} \otimes_{K}\left(Q\left(\varepsilon_{4 n}\right) / K, \alpha\right)$ is 2 if and only if $f(2) z$ is even.

This completes the proof of the lemma.

We will let $S(K)_{p}$ denote the $p$-primary part of $S(K)$, and $W(K, p)$ denote the roots of unity in $K$ with $p$-power order.

THEOREM 1. Let $p$ be a rational prime. Then $S(K)_{p}$ is generated by algebra classes which contain an algebra of the form $\left(Q\left(\varepsilon_{n q}\right) / K, \alpha\right)$ where the values of $\alpha$ are in $W\left(Q\left(\varepsilon_{n q}\right), p\right), q$ is either 4 or an odd prime, and $q$ does not divide $n$.

Proof. This is a refinement of Theorem 3 of [6]. In that theorem Janusz showed the following:

1. If $p$ is odd, or $p=2$ and 4 divides $n$, then $S(K)_{p}$ is generated by classes which contain algebras of the following types:

(a) $\left(Q\left(\varepsilon_{n q}\right) / K, \alpha\right)$, the values of $\alpha$ in $W\left(Q\left(\varepsilon_{n}\right), p\right)$ and $q$ a prime 
not dividing $n$.

(b) $\left(K\left(\varepsilon_{q r}\right) / K, \beta\right)$, the values of $\beta$ in $W(K, p)$ and $q$ and $r$ distinct primes not dividing $n$.

2. If $p=2$ and $n$ is odd, then $S(K)_{p}$ is generated by classes which contain an algebra of type $(b)$, or of type $\left(\mathrm{a}^{\prime}\right)\left(Q\left(\varepsilon_{4 n q}\right) / K, \alpha\right)$, the values of $\alpha$ in $W\left(Q\left(\varepsilon_{4}\right), 2\right)$ and $q$ an odd prime not dividing $n$.

In order to prove Theorem 1, we must look closely at algebras of types (b) and $\left(a^{\prime}\right)$.

Let $\operatorname{Gal}\left(K\left(\varepsilon_{q r}\right) / K\right)=\langle\sigma\rangle \times\langle\tau\rangle$ where $\langle\sigma\rangle=\operatorname{Gal}\left(K\left(\varepsilon_{q}\right) / K\right)$ and $\langle\tau\rangle=\operatorname{Gal}\left(K\left(\varepsilon_{r}\right) / K\right)$. Also let $\zeta$ be a $p^{d}$ th root of unity, the highest $p$-power root of unity in $K$. Consider the algebra

$$
\Delta_{q r}=\left(K\left(\varepsilon_{q r}\right) / K, \beta\right)=\sum K\left(\varepsilon_{q_{r}}\right) u_{r} \quad(\gamma \in\langle\sigma\rangle \times\langle\tau\rangle)
$$

where $u_{\sigma} u_{\tau}=\zeta^{x} u_{\tau} u_{\sigma}, u_{\sigma}^{q-1}=\zeta^{y}$, and $u_{\tau}^{r-1}=\zeta^{z}$. By [8, 11$]$, the only restrictions on $x, y$, and $z$ are $\left(\zeta^{z}\right)^{\sigma-1}=\left(\zeta^{x}\right)^{N(\tau)}$ and $\left(\zeta^{y}\right)^{--1}=\left(\zeta^{-x}\right)^{N(\sigma)}$ where $N(\phi)=1+\phi^{2}+\cdots+\phi^{|\phi|-1}$. However both $\sigma$ and $\tau$ fix $\zeta$, so we get that $p^{d}$ divides both $x(r-1)$ and $x(q-1)$.

Now $\Delta_{q r}$ can have nonzero invariant only at the primes of $K$ which divide $q$ and $r$. This is because these are the only primes ramified in $K\left(\varepsilon_{q r}\right) / K$.

Suppose that $q$ is odd. Let $\tau^{g}=\left[K\left(\varepsilon_{r}\right) / K, q\right]$, the Frobenius automorphism of $q$ in $K\left(\varepsilon_{r}\right) / K$, and set $t=q^{f(q)}-1$. We have that

$$
\left(\frac{\beta\left(\sigma, \tau^{g}\right)}{\beta\left(\tau^{g}, \sigma\right)}\right)^{(q-1) / t} u_{\sigma}^{q-1}=\left(\varepsilon_{t}\right)^{\mu \nu}
$$

where $\mu=(q-1) / p^{d}$ and $\nu=x g+y(t /(q-1))$.

The inertia group of $q$ in $K\left(\varepsilon_{q r}\right) / K$ is $\langle\sigma\rangle$, so [7, Thm 3] implies that the $q$-local index of $\Delta_{q_{r}}$ is $\max \left\{p^{d-s}, 1\right\}$ where $p^{s}$ exactly divides $\nu$.

Now suppose that $p^{a}$ exactly divides $f(q)$. Then $p^{a}$ divides $g$ since $\left[K\left(\varepsilon_{r}\right) / K, q\right]=\left[K\left(\varepsilon_{r}\right) / Q, q\right]^{f(q)}$. Moreover, if $p=2, f(q)$ is even, and $q \equiv 3 \bmod 4$, then $2^{a+1}$ exactly divides $t /(q-1)$, otherwise $p^{a}$ exactly divides $t /(q-1)$. In the case where $p=2, f(q)$ is even and $q \equiv 3 \bmod 4$, we either have $2^{d}>2$ so that $x$ is even, or $2^{d}=2$ so that $\Delta_{q r}$ has $q$-local index 1 .

Hence in all cases, $\max \left\{p^{d-s}, 1\right\}$ takes its highest possible value when $p^{s}$ exactly divides $t /(q-1)$.

Now consider the algebra $\left(K\left(\varepsilon_{q}\right), \sigma, \zeta\right)$. Applying [7, Thm. 3] we see that the $q$-local index is $\max \left\{p^{d-c}, 1\right\}$ where $p^{c}$ exactly divides $t /(q-1)$. Further, the local index of $\left(K\left(\varepsilon_{q}\right), \sigma, \zeta\right)$ at any prime unequal to $q$ is 1 . Note that $\left(K\left(\varepsilon_{q}\right), \sigma, \zeta\right)$ inflated to $Q\left(\varepsilon_{n q}\right) / K$ has the form described in Theorem 1 .

If $r$ is even, then $K\left(\varepsilon_{q_{r}}\right)=K\left(\varepsilon_{q}\right)$ so that the $r$-local index of $\Delta_{q_{r}}$ 
is 1. Thus, in this case, some power of $\left(K\left(\varepsilon_{q}\right), \sigma, \zeta\right)$ has exactly the same set of invariants as $\Delta_{q r}$.

If $r$ is odd, then we may replace $q$ by $r$ in the above argument. Hence, some power of $\left(K\left(\varepsilon_{r}\right), \tau, \zeta\right)$ has the same invariants at primes dividing $r$ as $\Delta_{q r}$ does, and some power of $\left(K\left(\varepsilon_{q}\right), \sigma, \zeta\right)$ has the same invariants as $A_{q r}$ at primes dividing $q$.

Thus $\left[\Delta_{q r}\right]$ is contained in the group generated by the classes described in the theorem.

Now suppose that $p=2$ and $n$ is odd. Let $G=\operatorname{Gal}\left(Q\left(\varepsilon_{n}\right) / K\right)$ be given by the direct product

$$
G=\left\langle\phi_{1}\right\rangle \times\left\langle\phi_{2}\right\rangle \times \cdots \times\left\langle\phi_{k}\right\rangle
$$

where $\left\langle\phi_{i}\right\rangle$ has order $n_{i}$. Further, set $\langle\rho\rangle=\operatorname{Gal}\left(Q\left(\varepsilon_{4 n}\right) / Q\left(\varepsilon_{n}\right)\right)$ and $\langle\sigma\rangle=\operatorname{Gal}\left(Q\left(\varepsilon_{n q}\right) / Q\left(\varepsilon_{n}\right)\right)$, were $q$ is an odd prime not dividing $n$. Let $\zeta$ be a primitive fourth root of unity.

Consider the algebra

$$
\Delta_{2 q}=\left(Q\left(\varepsilon_{4 n q}\right) / K, \alpha\right)=\sum Q\left(\varepsilon_{4 n q}\right) u_{\gamma}
$$

where

$$
\begin{aligned}
u_{\rho} u_{\sigma} & =\zeta^{x_{0}} u_{\sigma} u_{\rho}, \quad u_{\rho} u_{\phi_{2}}=\zeta^{x_{i}} u_{\dot{\phi}_{i}} u_{\rho}, \\
u_{\sigma} u_{\phi_{i}} & =\zeta^{y_{i}} u_{\dot{\phi}_{i}} u_{\sigma}, \quad u_{\dot{\phi}_{i}} u_{\phi_{j}}=\zeta^{y_{i j}} u_{\dot{\phi}_{j}} u_{\phi_{i}}, \\
u_{\rho}^{2} & =\zeta^{z}, \quad u_{\sigma}^{q-1}=\zeta^{z_{0}}, \quad u_{\dot{\psi}_{i}}^{n_{i}}=\zeta^{z_{i}},
\end{aligned}
$$

for $i, j=1,2, \cdots, k$ and $i \neq j$. The conditions in [8, $\S 1]$ imply that

$z, y_{i}$, and $y_{i j}$ are even for $i, j=1,2, \cdots, k$ and $i \neq j$,

$2 z_{0} \equiv x_{0}(q-1) \bmod 4$,

$2 z_{i} \equiv x_{i} n_{i} \bmod 4$ for $i=1,2, \cdots, k$.

We have that $A_{2 q}$ can have nonzero invariants only at those primes of $K$ which divide $2, q$, or some prime which ramifies in $Q\left(\varepsilon_{n}\right) / K$. Moreover, the invariants of $A_{2 q}$ can only be 0 or $1 / 2$ since the only 2-power roots of unity in $K$ are $\{ \pm 1\}$.

Let

$$
\Delta_{q}=\left(Q\left(\varepsilon_{n q}\right) / K, \gamma\right)=\sum Q\left(\varepsilon_{n q}\right) v_{\tau}
$$

be the algebra such that

$$
\begin{aligned}
v_{\sigma} v_{\phi_{i}} & =\zeta^{y_{i}} v_{\phi_{i}} v_{\sigma}, \quad v_{\phi_{i}} v_{\phi_{j}}=v_{\dot{\phi}_{j}} v_{\phi_{i}}, \\
v_{\sigma}^{q-1} & =\zeta^{z_{0}^{*}}, \quad v_{\phi_{i}}^{n_{i}}=1,
\end{aligned}
$$

for $i, j=1,2, \cdots, k$ where 


$$
\begin{array}{lll}
z_{0} & \text { if } & q \equiv 1 \bmod 4 \\
z_{0}^{*}=0 & \text { if } & q \equiv 3 \bmod 4 \text { and } f(q) \text { is even } \\
z_{0}+x_{0} r & \text { if } & q \equiv 3 \bmod 4 \text { and } f(q) \text { is odd }
\end{array}
$$

where

$$
r^{-1} \equiv \frac{q^{f(q)}-1}{q-1} \bmod 4
$$

Note that the $y_{i}$ are all even, and that $z_{0}+x_{0} r$ is even when $q \equiv 3$ $\bmod 4$ and $f(q)$ is odd. Thus the values of $\gamma$ are all +1 or -1 , and $\Delta_{q}$ is in $S(K)$.

Further, let

$$
\Delta_{2}=\left(Q\left(\varepsilon_{4 n}\right) / K, \gamma^{\prime}\right)=\sum Q\left(\varepsilon_{4 n}\right) w_{\tau}
$$

be the algebra such that

$$
\begin{aligned}
w_{\rho} w_{\phi_{i}} & =\zeta^{x_{i}} w_{\phi_{i}} w_{\rho}, \quad w_{\phi_{i}} w_{\phi_{j}}=\zeta^{y_{i j}} w_{\phi_{j}} w_{\phi_{i}}, \\
w_{\rho}^{2} & =\zeta^{z^{*}}, \quad w_{\phi_{i}}^{n_{i}}=\zeta^{z_{i}}
\end{aligned}
$$

for $i, j=1,2, \cdots, k$ and $i \neq j$ where

$$
\begin{aligned}
z^{*} & =z+x_{0} & & \text { if } \\
& =z & & \text { otherwise }
\end{aligned}
$$

Observe that both $\Delta_{q}$ and $\Delta_{2}$ belong to classes of the type described in the theorem.

Claim. The algebra $\Delta_{2 q}$ is equivalent to $\Delta_{2} \otimes_{K} \Delta_{q}$ in $B(K)$.

Proof of Claim. We will show that $\Delta_{2 q}$ and $\Delta_{2} \otimes \Delta_{q}$ have the same set of invariants. This is the same as showing that the local indices of these algebras are the same at $q, 2$, and the primes ramified in $Q\left(\varepsilon_{n}\right) / K$ because the invariants can be only 0 or $1 / 2$.

First consider the $q$-local indices of $\Delta_{2 q}$ and $\Delta_{2} \otimes \Delta_{q}$. Let the Frobenius automorphism for $q$ in $Q\left(\varepsilon_{4 n}\right) / K$ be $\eta_{q}=\rho^{g} \Pi \phi_{i}^{g i}$, and set $t=q^{f(q)}-1$. Then

$$
\left(\frac{\alpha\left(\sigma, \eta_{q}\right)}{\alpha\left(\eta_{q}, \sigma\right)}\right)^{(q-1) / t} u_{\sigma}^{q-1}=\left(\varepsilon_{t}\right)^{(q-1) \nu_{0} / 4}
$$

where

$$
\nu_{0}=g x_{0}+\mu \sum g_{i} y_{i}+z_{0}(t /(q-1))
$$

where 


$$
\begin{array}{ccc}
\mu=-1 & \text { if } & g=1 \\
=1 & \text { if } & g=0 .
\end{array}
$$

By [6, Thm. 3], the $q$-local index of $\Delta_{2 q}$ is given by

$$
\frac{q-1}{\left(\nu_{0}(q-1), q-1\right)}=\begin{array}{lll}
1 & \text { if } \quad \nu_{0} \equiv 0 \bmod Z \\
2 & \text { if } \quad \nu_{0} \equiv 1 / 2 \bmod Z .
\end{array}
$$

Now $q$ does not ramify in $Q\left(\varepsilon_{4 n}\right) / K$, so the $q$-local index of $\Delta_{2} \otimes \Delta_{q}$ is equal to the $q$-local index of $\Delta_{q}$.

The restriction of $\eta_{q}$ to $Q\left(\varepsilon_{n}\right)$ is the Frobenius automorphism of $q$ in $Q\left(\varepsilon_{n}\right) / K$; we will denote this by $\eta_{q}^{\prime}$.

We have that

$$
\left(\frac{\gamma\left(\sigma, \eta_{q}^{\prime}\right)}{\gamma\left(\eta_{q}^{\prime}, \sigma\right)}\right)^{(q-1) / t} v_{\sigma}^{q-1}=\left(\varepsilon_{t}\right)^{(q-1) \nu_{0}^{\prime}}
$$

where

$$
\nu_{0}^{\prime}=\frac{1}{4}\left[\sum g_{i} y_{i}+z_{0}^{*}(t /(q-1))\right]
$$

Hence the $q$-local index of $\Delta_{2} \otimes \Delta_{q}$ is given by

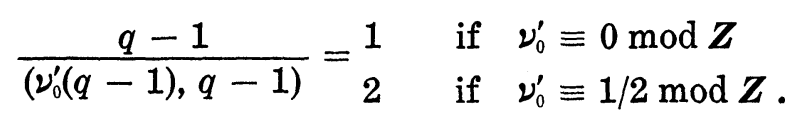

Now if $q \equiv 1 \bmod 4$, then $g=0$ and $z_{0}^{*}=z_{0}$, so $\nu_{0}=\nu_{0}^{\prime}$ and $\Delta_{2 q}$ has the same $q$-local index as $\Delta_{2} \otimes \Delta_{q}$. If $q \equiv 3 \bmod 4$ and $f(q)$ is even, then $g=0$ and 4 divides $t /(q-1)$, so that $\nu^{\prime} \equiv \nu_{0}^{\prime} \bmod Z$. Thus again $\Delta_{2 q}$ and $\Delta_{2} \otimes_{K} \Delta_{q}$ have the same $q$-local index. Finaliy suppose that $q \equiv 3 \bmod 4$ and $f(q)$ is odd. In this case $g=1$ so that

$$
g x_{0}+z_{0}(t /(q-1)) \equiv z_{0}^{*}(t /(q-1)) \bmod 4 .
$$

Hence $\nu_{0} \equiv \nu_{0}^{\prime} \bmod \boldsymbol{Z}$ and $\Delta_{2 q}$ has the same $q$-local index as $\Delta_{2} \otimes \Delta_{q}$.

Now let $l$ be a prime which ramifies in $Q\left(\varepsilon_{n}\right) / K$. We will compare the $l$-local indices of $\Delta_{2 q}$ and $\Delta_{2} \otimes \Delta_{q}$. Let $\langle\omega\rangle$ be the inertia group of $l$ in $Q\left(\varepsilon_{n}\right) / K$ where $\omega=\Pi \phi_{i}^{a_{i}}$, and let $\eta_{l}=\rho^{g} \sigma^{g_{0}} \Pi \phi_{i}^{g_{i}}$ be a Frobenius automorphism of $l$ in $Q\left(\varepsilon_{4 n q}\right) / K$. Then $\eta_{l}^{\prime}=\rho^{g} \Pi \phi_{i}^{g_{i}}$ and $\eta_{l}^{\prime \prime}=\sigma^{g_{0}} \Pi \phi_{i}^{g_{i}}$ are Frobenius automorphisms of $l$ in $Q\left(\varepsilon_{i_{n}}\right) / K$ and $Q\left(\varepsilon_{n q}\right) / K$ respectively. Let $e$ be the ramification index of $l$ in $Q\left(\varepsilon_{n}\right) / K$. Then we have $v_{\omega}^{e}=1$ and $w_{\omega}^{e}=u_{\omega}^{e}$. Moreover

$$
\frac{\alpha\left(\omega, \eta_{l}^{\prime \prime}\right)}{\alpha\left(\eta_{l}^{\prime \prime}, \omega\right)}=\frac{\gamma\left(\omega, \eta_{l}^{\prime \prime}\right)}{\gamma\left(\eta_{l}^{\prime \prime}, \omega\right)} \frac{\gamma^{\prime}\left(\omega, \eta_{l}^{\prime}\right)}{\gamma^{\prime}\left(\eta_{l}^{\prime}, \omega\right)}
$$

Hence, by [7, Thm. 3], we see that $\Delta_{2 q}$ and $\Delta_{2} \otimes \Delta_{q}$ have the same 


\section{$l$-local index.}

Finally, we must compare the 2-local indices of $A_{2 q}$ and $A_{2} \otimes \Delta_{q}$. Let $\sigma^{g_{0}} \Pi \dot{\phi}_{i}^{g_{i}}$ be the Frobenius automorphism of 2 in $Q\left(\varepsilon_{n q}\right) / K$, then Lemma 1 implies that the 2-local index of $A_{2 q}$ is 2 if and only if $\nu=g_{0} x_{0}+\sum g_{i} x_{i}+(z / 2) f(2)$ is odd. Further, the 2-local index of $\Delta_{2} \otimes_{K} \Delta_{q}$, which is the 2-local index of $A_{2}$, is 2 if and only if $\nu^{\prime}=\sum x_{i} g_{i}+\left(z^{*} / 2\right) f(2)$ is odd.

If $f(2)$ is even, then $g_{0}$ is even since

$$
\left[Q\left(\varepsilon_{n q}\right) / K, 2\right]=\left[Q\left(\varepsilon_{n q}\right) / Q, 2\right]^{f(2)} \text {. }
$$

Thus $\nu \equiv \nu^{\prime} \bmod 2$ and $\Delta_{2 q}$ has the same 2-local index as $\Delta_{2} \otimes \Delta_{q}$. If $f(2)$ is odd and $q \equiv 1$ or $7 \bmod 8$, then 2 is a square modulo $q$, so that $g$ must be even. Hence, once again $\nu \equiv \nu^{\prime} \bmod 2$ and $\Delta_{2 q}$ and $\Delta_{2} \otimes \Delta_{q}$ have the same 2-local index. Finally suppose that $f(2)$ is odd and that $q \equiv 3$ or $5 \bmod 8$. Then $g$ is odd and $z^{*}=z_{0}+x_{0}$, so $g x_{0}+(z / 2) f(2)$ is equivalent to $\left(z^{*} / 2\right) f(2)$ modulo 2 . Thus again $\nu \equiv \nu^{\prime}$ $\bmod 2$.

This completes the proof of the claim and of the theorem.

3. $S(K)_{2}$ when $Q\left(\varepsilon_{n}\right) / K$ is cyclic. In this section we will completely chararacterize the classes in $S(K)_{2}$ by the behavior of of their invariants in the case where $\mathrm{Gal}(L / K)$ is cyclic. Before beginning these calculations we need to prove the following lemma.

LEMma 2. Suppose that $K \subset F$ are subfields of a cyclotomic field and that $[F: K]$ is not divisible by the rational prime $p$. If there are no p-power roots of unity in $F$ which are not in $K$, then $S(F)_{p}=F \otimes_{k} S(K)_{p}$.

Proof. Clearly $S(F)_{p} \supseteqq F \bigotimes_{k} S(K)_{p}$. We need to show containment in the other direction.

Let $L$ be the smallest cyclotomic field containing $F$, and let $G=\operatorname{Gal}(L / K)$ be given by

$$
G=\prod_{i=1}^{t}\left\langle\phi_{i}\right\rangle \times \prod_{j=1}^{s}\left\langle\dot{\psi}_{j}\right\rangle
$$

where the order of each $\left\langle\dot{\phi}_{i}\right\rangle$ is a power of $p$ and the order of each $\left\langle\psi_{j}\right\rangle, n_{j}$, is relatively prime to $p$. It follows that $H=\mathrm{Gal}(L / K)$ is given by

$$
H=\prod_{\imath=1}^{t}\left\langle\dot{\phi}_{i}\right\rangle \times \prod_{j=1}^{s^{\prime}}\left\langle\psi_{j}^{\prime}\right\rangle
$$

where $\prod_{j=1}^{s}\left\langle\psi_{j}\right\rangle$ is a subgroup of $\prod_{j=1}^{s^{\prime}}\left\langle\psi_{j}^{\prime}\right\rangle$.

By Theorem 1, $S(F)_{p}$ is generated by classes containing algebras 
of the form

$$
\left(L\left(\varepsilon_{q}\right) / F, \alpha\right)=\sum_{\sigma} L\left(\varepsilon_{q}\right) U_{\sigma}
$$

where $q$ is either 4 or an odd prime and the values of $\alpha$ are $p$-power roots of unity.

Suppose that $U_{\psi_{j}}^{n}=\zeta^{z_{j}}$ where $\zeta$ is a primitive $p^{d}$ th root of unity. The order of $\psi_{j}$ is prime to $p$, so $\psi_{j}(\zeta)=\zeta$ unless $\zeta$ is not in $F$, in which case $S(F)_{p}=F \otimes_{K} S(K)_{p}=1$. Set $\gamma=-z_{j} / n_{j}$ modulo $p^{d}$. Now replace $U_{\psi_{j}}$ by $\zeta^{\lambda} U_{\psi_{j}}$ in $\left(L\left(\varepsilon_{q}\right) / F, \alpha\right)$. This gives an equivalent algebra, but now

$$
\left(\zeta^{\lambda} U_{\psi_{j}}\right)^{n_{j}}=\zeta^{0}=1 .
$$

Hence we might as well have started with $z_{j}=0$ for $j=1,2, \cdots, s$.

Now suppose that $U_{\psi_{j}} U_{\tau}=\zeta^{x_{j}} U_{\tau} U_{\psi_{j}}$ for some $\tau$ in $\operatorname{Gal}\left(L\left(\varepsilon_{q}\right) / F\right)$, $\tau$ not in $\left\langle\psi_{j}\right\rangle$. Then

$$
\begin{aligned}
1=U_{\psi_{j}}^{n}=\left(U_{\tau}^{-1} U_{\psi_{j}} U_{\tau}\right)^{n_{j}} & =\prod_{\tau=0}^{n_{j}-1} \psi_{j}^{i}\left(\zeta^{x_{j}}\right) \\
& =\zeta^{n_{j} x_{j}} .
\end{aligned}
$$

However $n_{j}$ is prime to $p$, so $x_{j}$ must be 0 . Thus $U_{\psi_{j}} U_{\tau}=U_{\tau} U_{\psi_{j}}$ for all $\tau \in \operatorname{Gal}\left(L\left(\varepsilon_{q}\right) / F\right)$. This is true for all $\psi_{j}, j=1,2, \cdots, s$.

Therefore

$$
\left[\left(L\left(\varepsilon_{q}\right) / F, \alpha\right)\right]=\left[\left(E_{1} / F, \alpha_{1}\right) \bigotimes_{F}\left(E_{2} / F, \alpha_{2}\right)\right]
$$

where $E_{1}$ is the field fixed by $\Pi_{i=1}^{t}\left\langle\phi_{i}\right\rangle$ and $E_{2}$ is the field fixed by $\Pi_{j=1}^{s}\left\langle\psi_{j}\right\rangle$. Moreover $\alpha_{1}$ is the trivial factor set, so $\left[\left(E_{1} / F, \alpha_{1}\right)\right]=[F]$.

Further, $\left[\left(E_{2} / F, \alpha_{2}\right)\right]=\left[F \otimes_{K}\left(E_{2} / K, \alpha_{2}^{\prime}\right)\right]$ where $\alpha_{2}^{\prime}$ restricted to $\Pi_{i=1}^{t}\left\langle\dot{\phi}_{i}\right\rangle$ equals $\alpha_{2}$ and $\alpha_{2}^{\prime}$ is trivial on Gal $(F / K)$. This makes $\alpha_{2}^{\prime}$ a factor set by the same reasoning we used to ascertain that $\alpha$ is equivalent to a factor set with nontrivial values only on $\prod_{i=1}^{t}\left\langle\phi_{i}\right\rangle$.

This completes the proof of the lemma.

Notice that this lemma implies that an algebra class [A] in $S(F)_{p}$ has $q_{i}$-local index $p^{a_{i}}$ for some sets of primes $q_{1}, \cdots, q_{t}$ if and only if there is an algebra class $[D]$ in $S(K)_{p}$ with exactly the same local indices. Hence, if we can find the possible local indices for classes in $S(F)_{p}$, then we have found them for classes in $S(K)_{p}$.

In the following theorems we assume that $[K: Q]$ is even. We may do this because $S(K)$ consists of all classes in $B(K)$ with uniformly distributed invariants of value 0 or $1 / 2$ if $[K: Q]$ is odd. This follows from [2].

A. $S(K)_{2}$ when $n$ is odd.

THEOREM 2. Let $K$ be a field contained in $L=Q\left(\varepsilon_{n}\right)$ where $n$ 
is odd such that $\mathrm{Gal}(L / K)$ is cyclic and [K:Q] is even. Then the 2-primary part of $S(K)$ consists of those classes $[A]$ in $B(K)$ with uniformly distributed invariants of value 0 or $1 / 2$ which satisfy the following conditions.

( I ) For a prime $p$ which divides $n$, $\operatorname{inv}_{p}[A]=0$ if $e(P)$ is odd or if $[L: K] / e(P)$ is even.

(II) For any prime $q$, $\operatorname{inv}_{q}[A]=0$ if $f(q)$ is even and a Frobenius automorphism of $q$ is a square in $\mathrm{Gal}(L / K)$.

(III) Let $p$ be a prime which divides $n$ to which (I) does not apply. Suppose that $f(p)$ is odd and that $|(p-1) / e(p)|_{2} \geqq\left|p^{\prime}-1\right|_{2}$ for every prime $p^{\prime}$ which divides $n$ and is unequal to $p$. Then the invariant of $[A]$ is $1 / 2$ at an even number of primes in the set

$$
\{p\} \cup\{\text { primes } q:(q / p)=-1 \text { and }(q, n)=1\}
$$

where $(q / p)$ is the Legendre symbol.

Proof. Let $G=\operatorname{Gal}(L / K)$ be $\langle\phi\rangle$ and have order $m=2^{c} c^{\prime}$, $\left(2, c^{\prime}\right)=1$.

Step 1. We need to determine the invariants of the generators of $S(K)_{2}$ given in Theorem 1.

(a) Let $\Delta_{q}=\Delta_{q}(x, y, z)$ be an algebra

$$
\Delta_{q}=\left(L\left(\varepsilon_{q}\right) / K, \alpha\right)=\sum_{\tau} L\left(\varepsilon_{q}\right) U_{\tau}
$$

where $q$ is an odd prime not dividing $n$ and the values of $\alpha$ are in $\{ \pm 1\}$. Let $\langle\gamma\rangle=\operatorname{Gal}\left(L\left(\varepsilon_{q}\right) / L\right)$. Then the factor set $\alpha$ is determined by the integers $x, y$, and $z$ where

$$
\begin{aligned}
U_{r} U_{\phi} & =(-1)^{x} U_{\phi} U_{r}, \\
\left(U_{r}\right)^{q-1} & =(-1)^{y}, \\
\left(U_{\phi}\right)^{m} & =(-1)^{z} .
\end{aligned}
$$

The restrictions given in $[8, \S 1]$ reduce to:

$$
x=0 \text { if } m \text { is odd . }
$$

Suppose that the Frobenius automorphism of $q$ in $L / K$ is $\phi^{g}$. Set $t(q)=q^{f(q)}-1$. Then

$$
\left(\frac{\alpha\left(\gamma, \phi^{g}\right)}{\alpha\left(\phi^{g}, \gamma\right)}\right)^{(q-1) / t(q)} U_{r}^{q-1}=\left(\varepsilon_{t(q)}\right)^{((q-1) / 2) \nu}
$$

where $\nu=x g+y(t(q) /(q-1))$. The inertia group of $q$ in $L\left(\varepsilon_{q}\right) / K$ is $\langle\gamma\rangle$, so $[8, \mathrm{Thm} .3]$ implies that the $q$-local index of $\left[\Delta_{q}\right]$ is given by 


$$
\begin{aligned}
\frac{q-1}{(\nu(q-1) / 2, q-1)} & =1 & & \text { if } \nu \text { is even } \\
& =2 & & \text { if } \nu \text { is odd } .
\end{aligned}
$$

Now $t(q) /(q-1)$ is odd if and only if $f(q)$ is odd, so we get

$$
\operatorname{inv}_{q}\left[\Delta_{q}\right]=1 / 2 \Longleftrightarrow x g+y f(q) \quad \text { is odd . }
$$

Now suppose that $p$ divides $n$. Let $\gamma^{h} \phi^{h^{\prime}}$ be a Frobenius automorphism for $p$ in $L\left(\varepsilon_{q}\right) / K$, and let $\left\langle\phi^{a}\right\rangle$ be the inertia group of $p$ in $L\left(\varepsilon_{q}\right) / K$. Then

$$
\left(\frac{\alpha\left(\phi^{a}, \gamma^{h} \phi^{h^{\prime}}\right)}{\alpha\left(\gamma^{h} \phi^{h^{\prime}}, \phi^{a}\right)}\right)^{e(p) / t(p)}\left(U_{\phi}^{a}\right)^{e(p)}=\left(\varepsilon_{t(p)}\right)^{(e(p) / 2) \nu^{\prime}}
$$

where $\nu^{\prime}=x a h+\mu z(t(p) / e(p))$,

where

$$
\begin{array}{rll}
\mu=0 & \text { if } & a=0 \\
=1 & \text { if } & a \neq 0 .
\end{array}
$$

Thus the $p$-local index of $\left[\Delta_{q}\right]$ is given by

$$
\begin{aligned}
\frac{e(p)}{\left(\nu^{\prime} e(p) / 2, e(p)\right)} & =1 & & \text { if } \nu^{\prime} \text { is even } \\
& =2 & & \text { if } \nu^{\prime} \text { is odd } .
\end{aligned}
$$

Hence

$$
\operatorname{inv}_{p}\left[\Delta_{q}\right]=1 / 2 \Leftrightarrow x a h+\mu z\left(\frac{t(p)}{e(p)}\right) \quad \text { is odd . }
$$

(b) Let $\Delta_{2}=\Delta_{2}(x, y, z)$ be the algebra

$$
\Delta_{2}=\left(L\left(\varepsilon_{4}\right) / K, \alpha\right)=\sum_{\tau} L\left(\varepsilon_{4}\right) U_{\tau}
$$

where the values of $\alpha$ are in $\left\{ \pm 1, \pm \varepsilon_{4}\right\}$. If $\langle\rho\rangle=\operatorname{Gal}\left(L\left(\varepsilon_{4}\right) / L\right)$, then the factor set $\alpha$ is determined by the integers $x, y$, and $z$ where

$$
\begin{aligned}
U_{\rho} U_{\phi} & =\left(\varepsilon_{4}\right)^{x} U_{\phi} U_{\rho}, \\
\left(U_{\rho}\right)^{2} & =\left(\varepsilon_{4}\right)^{y}, \\
\left(U_{\phi}\right)^{m} & =\left(\varepsilon_{4}\right)^{z} .
\end{aligned}
$$

The restrictions on $x, y$, and $z$ are

$y$ is even

$$
x m+2 z \equiv 0 \bmod 4 \text {. }
$$

Let $[L / K, 2]=\phi^{g}$. Then by Lemma 1 , 


$$
\operatorname{inv}_{2}\left[\Delta_{2}\right]=1 / 2 \Leftrightarrow x y+(y / 2) f(2) \quad \text { is odd . }
$$

Now let $p$ be a prime dividing $n$. Let $\rho^{k} \phi^{k^{\prime}}$ be a Frobenius automorphism of $p$ in $L\left(\varepsilon_{4}\right) / K$, and let $\left\langle\dot{\varphi}^{a}\right\rangle$ be the inertia group of $p$ in $L / K$. Then

$$
\left(\frac{\alpha\left(\dot{\phi}^{a}, \rho^{k} \dot{\phi}^{k^{\prime}}\right)}{\alpha\left(\rho^{k} \dot{\phi}^{k^{\prime}}, \dot{\rho}^{\alpha}\right)}\right)^{e(p) / t(p)}\left(U_{\phi}^{a}\right)^{e(p)}=\left(\varepsilon_{t(\rho)}\right)^{(\ell(p) / 4) \nu^{\prime \prime}}
$$

where

where

$$
\begin{array}{rlrl}
\nu^{\prime \prime}=x a k+\mu z\left(\frac{t(p)}{e(p)}\right) & \\
\mu=0 & \text { if } & a=0 \\
=1 & \text { if } & a \neq 0 .
\end{array}
$$

Thus

$$
\operatorname{inv}_{p}\left[A_{2}\right]=1 / 2 \Leftrightarrow \frac{x a k}{2}+\frac{\mu z}{2}\left(\frac{t(p)}{e(p)}\right) \quad \text { is odd . }
$$

Finally observe that if $l$ is a finite prime which does not divide $n q$, then $l$ does not ramify in $L\left(\varepsilon_{q}\right) / K$ and so $\operatorname{inv}_{l}\left[\Delta_{q}\right]=0$.

Now assume that $[L: K]$ is odd. Then $S(K)_{2}=K \otimes_{Q} S(Q)$ by [5, Cor. 2]. This means that there is an algebra class [A] in $S(K)_{2}$ with $\operatorname{inv}_{q}[A]=1 / 2$ if and only if the order of the decomposition group of $q$ in $K / Q, f(q) e(q, K / Q)$, is odd.

For each prime $p$ which divides $n$, we must have that $e(p, K / Q)$ is even and $e(p)$ is odd. Thus condition (I) of the theorem applies, and is satisfied. Further, every element in $\mathrm{Gal}(L / K)$ is a square, so condition (II) reduces to: For any prime $q$, $\operatorname{inv}_{q}[A]=0$ if $f(q)$ is even. Hence this condition is satisfied. Condition (III) is trivially satisfied since condition (I) applies to each prime $p$ which divides $n$.

Suppose now that $q$ is a prime not dividing $n$ such that $f(q)$ is odd. Then the decomposition group of $q$ in $K / Q$ has odd order. Thus the algebra $K \otimes_{Q}\left(Q\left(\varepsilon_{q^{\prime}}\right), \gamma,-1\right)$ has invariant $1 / 2$ at $q$ and invariant 0 elsewhere, where $\langle\gamma\rangle=\operatorname{Gal}\left(Q\left(\varepsilon_{q^{\prime}}\right) / Q\right)$ and $q^{\prime}=q$ unless $q$ is even, in which case $q^{\prime}=4$. Note that $K$ cannot be a real field in this case, so that the invariants of any algebra in $B(K)$ are 0 at the infinite primes of $K$.

We have now shown that the theorem holds if $[L: K]$ is odd. For the rest of the proof we shall assume that $[L: K]$ is even. By Lemma 2, we may assume that $[L: K]=2^{c}$ for $c \geqq 1$.

Suppose that $K$ is a real field. Pick a prime $p$ such that $f(p) e(p, K / Q)$ is even. This can always be done since $[K: Q]$ is assumed to be even. Consider the algebra $K \otimes_{Q} D_{p}$ where $\left[D_{p}\right] \in S(Q)$ has invariant $1 / 2$ only at $p$ and the infinite prime $p_{\infty}$. Then $\left[K \otimes D_{p}\right]$ 
has invariant $1 / 2$ just at the infinite primes of $K$. Hence $\Omega\left(p_{\infty}\right)$ is in $K$. This settles the case with respect to the infinite primes since $B(C)=\{1\}$ where $C$ is the complex numbers. For the remainder of the proof, "prime" will mean "finite prime."

Step 2. Condition (I) is satisfied.

Suppose that $p$ is a prime which divides $n$, and that $e(p) \neq 2^{t}$. Then $a$ is even where $\left\langle\phi^{a}\right\rangle$ is the invertia group of $p$ in $L / K$. Hence $(p-1) / e(p)$ is even because it is divisible by $a$ if $e(p) \neq 1$. Thus (3.2) implies that $\operatorname{inv}_{p}\left[\Delta_{q}\right]=0$ for all odd primes $q$ which do not divide $n$. Now consider $\Delta_{2}$. If $a=0$, then (3.5) implies that $\operatorname{inv}_{p}\left[\Delta_{2}\right]=0$ since $\mu=0$. If $a \neq 0$, then $2^{t} \geqq 4$ so that $p \equiv 1 \bmod 4$. Hence $\left[Q\left(\varepsilon_{4}\right) / Q, p\right]=1$, so in (3.5) we have that $k=0$. Moreover, (3.3) implies that $z$ is even, so $\operatorname{inv}_{p}\left[\Delta_{2}\right]=0$.

We have shown that each of the generators of $S(K)_{2}$ has 0 invariant at $p$. Hence $\operatorname{inv}_{p}[A]=0$ for all $[A]$ in $S(K)_{2}$ and condition (I) is satisfied.

\section{Step 3. Condition (II) is satisfied.}

Suppose that $p$ is a prime dividing $n$ such that $f(p)$ is even and condition (I) does not apply to $p$. Note that the identity element in $\mathrm{Gal}(L / K)$ is a Frobenius automorphism for $p$ in $L / K$ in this case, so condition (II) does apply to $p$.

Observe that $t(p) / e(p)$ is even, and in the case where $e(p)=2$, $t(p) / e(p)$ is divisible by 4 . This is so because $f(p)$ is even and $e(p)=2^{t}$ must divide $p-1$.

Let $l$ be either 4 or an odd prime not dividing $n$, and suppose that $\gamma^{h}$ is a Frobenius automorphism for $p$ in $L\left(\varepsilon_{l}\right) / K$ where $\langle\gamma\rangle=$ $\operatorname{Gal}\left(L\left(\varepsilon_{l}\right) / L\right)$. If $l$ is an odd prime then $h$ must be even since $f(p)$ is even. If $l=4$, then $h=0$. Further, by (3.3), $z$ is even when $e(p) \geqq 4$. Thus (3.2) and (3.5) imply that $\operatorname{inv}_{p}\left[\Delta_{l^{\prime}}\right]=0$ where $l^{\prime}=l$ if $l$ is odd or $l^{\prime}=2$ if $l=4$.

Hence, for $p$, condition (II) is satisfied on the generators of $S(K)_{2}$. Therefore condition (II) is satisfied for all primes which divide $n$.

Now suppose that $q$ is a prime which does not divide $n$ such that $f(q)$ is even and $[L / K, q]=\phi^{g}$ is a square in Gal $(L / K)$. Then $g$ is even so that $g x+f(q) y$, or $g x+f(q) y / 2$ in the case of $q=2$, is even for all permissible values of $x$ and $y$. Thus, by (3.1) and (3.4), $\operatorname{inv}_{q}\left[\Delta_{q}\right]=0$.

Classes of the type $\left[\Delta_{q}\right]$ are the only classes amongst the generating classes given by Theorem 1 which might possibly have 
nonzero invariant at primes of $K$ dividing $q$. Hence $\operatorname{inv}_{q}[A]=0$ for all $[A]$ in $S(K)_{2}$, and condition (II) is satisfied for primes which do not divide $n$.

Step 4. For each prime $l$ to which conditions (I) and (II) do not apply, there is a class $[A]$ in $S(K)_{2}$ such that $\operatorname{inv}_{l}[A]=1 / 2$.

First suppose that $q$ is a prime which does not divide $n$. If $f(q)$ is odd, then the algebra

$$
\begin{aligned}
\Delta_{q}^{0} & =\Delta_{q}(0,2,0) & \text { if } & q=2 \\
& =\Delta_{q}(0,1,0) & \text { if } & q \neq 2
\end{aligned}
$$

has invariant $1 / 2$ at $q$ and invariant 0 elsewhere. Hence $\Omega(q)=\left[\Delta_{q}^{0}\right]$ if $f(q)$ is odd.

Suppose that $f(q)$ is even and that $[L / K, q]=\phi^{g}$ where $g$ is odd. By (3.1) and (3.4), the algebra

$$
\begin{aligned}
\Delta_{q}^{1} & =\Delta_{q}(1,0,1) & & \text { if } q=2 \text { and } 2^{c}=2 \\
& =\Delta_{q}(1,0,0) & & \text { otherwise }
\end{aligned}
$$

has invariant $1 / 2$ at $q$.

Now let $p$ be a prime which divides $n$ such that neither condition (I) nor condition (II) applies to $p$. Hence, $f(p)$ is odd. Pick an odd prime $q$ not dividing $n$ such that $\left[Q\left(\varepsilon_{4 p}\right) / Q, q\right]=\psi$ where $\langle\psi\rangle=$ Gal $\left(Q\left(\varepsilon_{p}\right) / Q\right)$. There exist infinitely many such $q$ by the Tchebotarev density theorem. This choice of $q$ insures that $q \equiv 1 \bmod 4$ and that $(q / p)=-1$. Hence, by quadratic reciprocity, $(p / q)=-1$. Thus $h$ must be odd where $\gamma^{h}$ is a Frobenius automorphism of $p$ in $L\left(\varepsilon_{q}\right) / K$. Then by (3.2) $\operatorname{inv}_{p}\left[\Delta_{q}^{1}\right]=1 / 2$ where $\Delta_{q}^{1}$ is the algebra described above. This is because $a$ is odd if condition (I) does not apply.

Step 5. If condition (III) does not apply, then $\Omega(l)$ is in $S(K)_{2}$ for every prime $l$ to which conditions (I) and (II) do not apply.

Let $p$ be a prime which divides $n$ such that condition (I) does not apply to $p$. This means that $p$ is totally ramified in $L / K$. Hence $p$ is the only prime which is ramified in $L / K$, and so $p$ is the only prime dividing $n$ to which condition (I) does not apply.

Now suppose that condition (II) does not apply to $p$. We saw in Step 3 that this means that $f(p)$ is odd. Further suppose that $|(p-1) / e(p)|_{2}<\left|p^{\prime}-1\right|_{2}$ for some prime $p^{\prime} \neq p$ which divides $n$. Pick an odd prime $q_{0}$ which does not divide $n$ such that $\left[L\left(\varepsilon_{4}\right) / Q, q_{0}\right]=$ $\psi \psi^{\prime}$ where $\psi$ generates $\operatorname{Gal}\left(Q\left(\varepsilon_{p}\right) / Q\right)$ and $\psi^{\prime}$ generates $\operatorname{Gal}\left(Q\left(\varepsilon_{p^{\prime}}\right) / Q\right)$. Now $f\left(q_{0}\right)$ is divisible by the same power of 2 as $p^{\prime}-1$ is, hence $\left[L / K, q_{0}\right]=\phi^{g}$ where $g$ is even. Thus $\operatorname{inv}_{q_{0}}\left[\Delta_{q_{0}}^{1}\right]=0$. However our 
choice of $q_{0}$ insures that $q_{0} \equiv 1 \bmod 4$ and that $\left(q_{0} / p\right)=-1$. Thus the argument at the end of Step 3 gives $\operatorname{inv}_{p}\left[\Delta_{q_{0}}^{1}\right]=1 / 2$. Since $p$ is the only prime dividing $n$ at which $\Delta_{q_{0}}^{1}$ can have nonzero invariants, we have that $\Omega(p)=\left[\Delta_{q_{0}}^{1}\right]$.

Now let $q$ be a prime which does not divide $n$ such that condition (II) does not apply to $q$. We saw in Step 3 that $\Omega(q)$ is in $S(K)_{2}$ if $f(q)$ is odd. Further, if $f(q)$ is even, we have that $\operatorname{inv}_{q}\left[\Delta_{q}^{1}\right]=1 / 2$. Thus, if $\operatorname{inv}_{p}\left[\Delta_{q}^{1}\right]=0$, we have $\Omega(q)=\left[\Delta_{q}^{1}\right]$. If $\operatorname{inv}_{p}\left[\Delta_{q}^{1}\right]=1 / 2$, then $\Omega(q)=\left[\Delta_{q}^{1}\right] \bigotimes_{k} \Omega(p)$.

Step 6. Condition (III) is satisfied.

Let $p$ be a prime dividing $n$ to which condition (I) does not apply. Further suppose that $f(p)$ is odd and that $|(p-1) / e(p)|_{2} \geqq$ $\left|p^{\prime}-1\right|_{2}$ for every prime $p^{\prime} \neq p$ which divides $n$. This hypothesis, and the assumption that $[K: Q]$ is even, forces $p \equiv 1 \bmod 4$. We also have that $\langle\phi\rangle$ is the inertia group of $p$ in $L / K$.

Let $q$ be a prime not dividing $n$ such that $\operatorname{inv}_{p}\left[\Delta_{q}^{\prime}\right]=1 / 2$ where $\Delta_{q}^{\prime}$ is one of the generators of $S(K)_{2}$ given in Theorem 1. Let $[L / K, q]=\phi^{g}$ and let $\gamma^{h}$ be a Frobenius automorphism of $p$ in $L\left(\varepsilon_{q^{\prime}}\right) / K$ where $\langle\gamma\rangle=\operatorname{Gal}\left(L\left(\varepsilon_{q^{\prime}}\right) / K\right), q^{\prime}=q$ if $q$ is odd, and $q^{\prime}=4$ if $q=2$.

(a) Suppose that $q$ is odd. Then by (3.2), $h x$ must be odd. However, $h$ is odd if and only if $(p / q)=-1$ since $f(p)$ is odd. So, by the law of quadratic reciprocity, $(q / p)=-1$ and so $f(q)$ is divisible by the same power of 2 as $(p-1) / e(p)$ is. This implies that $g$ is odd. Hence $\operatorname{inv}_{q}\left[\Delta_{q}^{\prime}\right]=1 / 2$.

(b) Suppose that $q=2$. Then $h=0$ since $\left[Q\left(\varepsilon_{4}\right) / Q, p\right]=1$. Thus $z / 2(t(p) / e(p))$ must be odd. This means that $t(p) / e(p) \equiv 2 \bmod 4$ and $z$ is odd. By (3.3), this can only occur when $x$ is odd and $e(p)=2$. Thus $p \equiv 5 \bmod 8$, so that $(2 / p)=-1$. This implies that $f(2)$ is even and that $q$ is odd. Hence, by (3.4) $\operatorname{inv}_{2}\left[\Delta_{2}^{\prime}\right]=1 / 2$.

Now let $q$ be a prime not dividing $n$ such that $(q / p)=-1$ and $\operatorname{inv}_{q}\left[\Delta_{q}^{\prime \prime}\right]=1 / 2$ where $\Delta_{q}^{\prime \prime}$ is one of the algebras described in Theorem 1. Let $[L / K, q]=\phi^{g}$ and let $\gamma^{h}$ be a Frobenius automorphism of $p$ in $L\left(\varepsilon_{q^{\prime}}\right) / K$ where $\langle\gamma\rangle=\operatorname{Gal}\left(L\left(\varepsilon_{p^{\prime}}\right) / K\right)$ and $q^{\prime}=q$ if $q$ is odd or $q^{\prime}=4$ if $q=2$.

By (3.1) and (3.4), $x g$ is odd. If $q$ is odd, then $h$ is odd so that $\operatorname{inv}_{p}\left[\Delta_{q}^{\prime \prime}\right]=1 / 2$. So suppose that $q=2$. Then we must have $p \equiv 5 \bmod 8$. This implies that $t(p) / e(p) \equiv 2 \bmod 4$, and, by (3.3), that $z$ is odd. Hence (3.5) implies that $\operatorname{inv}_{p}\left[\Delta_{2}^{\prime \prime}\right]=1 / 2$.

We have now shown that

$$
\operatorname{inv}_{p}\left[\Delta_{q}\right]=1 / 2 \Leftrightarrow \operatorname{inv}_{q}\left[\Delta_{q}\right]=1 / 2 \text { and }(q / p)=-1 .
$$

Since every algebra class $[A]$ in $S(K)_{2}$ is generated by classes of 
this form, we have shown that condition (III) is satisfied.

Further, this proves that $\Omega(q)$ is in $S(K)_{2}$ if $(q / p)=1$ and condition (II) does not apply to $q$. This is because $\left[\Delta_{q}\right]$ can have nonzero invariants only at $p$ and $q$; we saw in Step 3 that we could arrange for nonzero invariants at $q$ and we have just seen that we cannot get nonzero invariants at $p$.

This completes the proof of the theorem.

B. $S(K)_{2}$ when $n$ is even.

Now suppose that $L=Q\left(\varepsilon_{n}\right)$ is a cyclotomic field containing $\zeta$, a primitive $2^{s}$ th root of unity for $s \geqq 2$. Further suppose that $K \subset L$ does not contain a fourth root of unity, and that $\operatorname{Gal}(L / K)=\langle\phi\rangle$ has order $2^{\circ} c^{\prime},\left(c^{\prime}, 2\right)=1$.

Let $\operatorname{Gal}(Q(\zeta) / Q)=\langle\rho\rangle \times\langle\psi\rangle$ where $\rho(\zeta)=\zeta^{-1}$ and $\psi(\zeta)=\zeta^{5}$. Then we may assume that $\phi=\rho \psi^{2^{r-2}} \tau$ where the order of $\left\langle\psi^{2 r-2}\right\rangle=$ $2^{s-r}$ divides the order of $\langle\tau\rangle$. Thus $\phi(\zeta)=\zeta^{-h}$ where $h=5^{2^{r-2}}$. We will keep this notation for the rest of this section.

We must determine the invariants of the generators of $S(K)_{2}$ given in Theorem 1 .

Let $\Delta_{q}=\Delta_{q}(x, y, z)$ be the algebra

$$
\Delta_{q}=\left(L\left(\varepsilon_{q}\right) / K, \alpha\right)=\sum_{\tau} L\left(\varepsilon_{q}\right) U_{\tau}
$$

where $q$ is a prime not dividing $n$ and the values of $\alpha$ are in $\langle\zeta\rangle$. Let $\langle\gamma\rangle=\operatorname{Gal}\left(L\left(\varepsilon_{q}\right) / L\right)$. The factor set $\alpha$ is determined by the integers $x, y$, and $z$ where

$$
\begin{aligned}
U_{\gamma} U_{\phi} & =\zeta^{x} U_{\phi} U_{r}, \\
U_{\gamma}^{q-1} & =\zeta^{y}, \\
U_{\phi}^{2^{c_{c^{\prime}}}} & =\zeta^{z} .
\end{aligned}
$$

The conditions in $[8, \S 1]$ require that

$$
\begin{aligned}
\zeta^{z}=\left(\zeta^{z}\right)^{\phi} & =\zeta^{-h z} \\
\left(\zeta^{y}\right)^{-h-1} & =\left(\zeta^{y}\right)^{\phi-1} \\
& =\left(\zeta^{-x}\right)^{N(r)} \\
& =\zeta^{-x(q-1)} \\
1=\left(\zeta^{z}\right)^{r-1} & =\left(\zeta^{x}\right)^{N(\phi)}
\end{aligned}
$$

where $N(\tau)=1+\tau^{2}+\cdots+\tau^{|\tau|-1}$ for a group element $\tau$. Hence

$$
2^{s-1} \text { divides } z \text {, }
$$

$$
y(h+1)-x(q-1) \equiv 0 \bmod 2^{s},
$$

2 divides $x$ if $c=s-r$. 
Now suppose that $[L / K, q]=\phi^{g}$. Then

$$
\left(\frac{\alpha\left(\gamma, \phi^{g}\right)}{\alpha\left(\phi^{g}, \gamma\right)}\right)^{(q-1) / t(q)} U_{\gamma}^{q-1}=\left(\varepsilon_{t(q)}\right)^{(q-1) \nu}
$$

where

$$
\nu=\frac{1}{2^{s}}\left[x\left(\frac{1-(-h)^{g}}{1+h}\right)+y\left(\frac{t(q)}{q-1}\right)\right] .
$$

Thus the $q$-local index of $\Delta_{q}$ is given by

$$
\begin{array}{rlrl}
\frac{q-1}{((q-1) \nu, q-1)} & =1 & & \text { if } \quad \nu \equiv 0 \bmod Z \\
& =2 \quad \text { if } \quad \nu \equiv 1 / 2 \bmod Z .
\end{array}
$$

Hence

$$
\operatorname{inv}_{q}\left[\Delta_{q}\right]=1 / 2 \Leftrightarrow \nu \equiv 1 / 2 \bmod Z \mathbf{Z} .
$$

Now suppose that $p$ is an odd prime which divides $n$. Let $\gamma^{b} \phi^{b^{\prime}}$ be a Frobenius automorphism of $p$ in $L\left(\varepsilon_{q}\right) / K$, and let $\left\langle\phi^{a}\right\rangle$ be the inertia group of $p$ in $L\left(\varepsilon_{q}\right) / K$.

Then

$$
\left(\frac{\alpha\left(\phi^{a}, \gamma^{b} \phi^{b^{\prime}}\right)}{\alpha\left(\gamma^{b} \phi^{b^{\prime}}, \phi^{a}\right)}\right)^{e(p) / t(p)}\left(U_{\phi^{a}}\right)^{e(p)}=\varepsilon_{t(p)}^{e(p) \nu}
$$

where

$$
\nu_{p}=\frac{1}{2^{s}}\left[x b\left(\frac{1-h^{a}}{1+h}\right)+\mu z\left(\frac{p^{f(p)}-1}{e(p)}\right)\right]
$$

where

$$
\begin{array}{rll}
\mu=0 & \text { if } & a=0 \\
=1 & \text { if } & a \neq 0 .
\end{array}
$$

Hence

$$
\operatorname{inv}_{p}\left[\Delta_{q}\right]=1 / 2 \Longleftrightarrow \nu_{p} \equiv 1 / 2 \bmod \boldsymbol{Z} .
$$

Finally suppose that 2 is ramified in $L / K$. Our assumption that the order of $\left\langle\psi^{2 r-2}\right\rangle$ divides the order of $\langle\tau\rangle$ implies that in this case $\operatorname{Gal}(L / K)=\langle\rho\rangle$.

Let $\eta=\gamma^{b}$ be a Frobenius automorphism of 2 in $L\left(\varepsilon_{q}\right) / K$. Let $f$ be the order of $\langle\eta\rangle$. We have

$$
\begin{aligned}
U_{\rho}\left(\left(1+\zeta^{x b}\right) U_{\eta}\right) & =\left(1+\zeta^{-x b}\right) U_{\rho} U_{\eta} \\
& =\left(1+\zeta^{-x b}\right) \zeta^{x b} U_{\eta} U_{\rho} \\
& =\left[\left(1+\zeta^{x b}\right) U_{\eta}\right] U_{\rho} .
\end{aligned}
$$


Let $\pi$ be a prime of $K$ which divides 2 . Then

$$
\begin{aligned}
K_{\pi} \otimes \Delta_{q} & =\sum_{i=0}^{1} \sum_{j=0}^{f-1} K_{\pi}\left(\varepsilon_{4}\right) K_{\pi}\left(\varepsilon_{q}\right) U_{\rho}^{i} U_{\eta}^{j} \\
& =\sum_{i=0}^{1} \sum_{j=0}^{f-1} K_{\pi}\left(\varepsilon_{4}\right) K_{\pi}\left(\varepsilon_{q}\right) U_{\rho}^{i}\left[\left(1+\zeta^{x b}\right) U_{\eta}\right]^{j} \\
& \cong \sum_{i=0}^{1} K_{\pi}\left(\varepsilon_{4}\right) U_{\rho}^{2} \bigotimes_{K_{\pi}} \sum_{j=0}^{f-1} K_{\pi}\left(\varepsilon_{q}\right)\left[\left(1+\zeta^{x b}\right) U_{\eta}\right]^{j} \\
& \cong\left(K_{\pi}\left(\varepsilon_{4}\right), \rho, U_{\rho}^{2}\right) \bigotimes_{K_{\pi}}\left(K_{\pi}\left(\varepsilon_{q}\right), \eta,\left[\left(1+\zeta^{x b}\right) U_{\eta}\right]^{f}\right) .
\end{aligned}
$$

Now $\left[\left(K_{\pi}\left(\varepsilon_{4}\right), \rho, U_{\rho}^{2}\right)\right]=K_{\pi} \otimes_{Q_{2}}\left(Q_{2}\left(\varepsilon_{4}\right), \rho, \zeta^{z}\right)$. Hence $\operatorname{inv}\left(K_{\pi}\left(\varepsilon_{4}\right), \rho, U_{\rho}^{2}\right)$ may be assumed to be 0 , since otherwise $e(2, K / Q)$ would be odd which would mean that $K=Q\left(\varepsilon_{n / 4}\right)$. The Schur subgroup of a cyclotomic field is given in [5].

Now let $V^{\prime}$ and $V$ be the exponential valuations of $K_{\pi}\left(\varepsilon_{4}\right)$ and $K_{\pi}$ respectively. Since $e\left(K_{\pi}\left(\varepsilon_{4}\right) / K\right)=2$, we have

$$
\begin{aligned}
V\left[\left(1+\zeta^{x b}\right) U_{\eta}\right]^{f} & =\frac{1}{2} V^{\prime}\left[\left(1+\zeta^{x b}\right) U_{\eta}\right]^{f} \\
& =\frac{1}{2}\left[V^{\prime}\left(1+\zeta^{x b}\right)^{f}+V^{\prime}\left(U_{\eta}^{f}\right)\right] \\
& =\frac{1}{2} f V^{\prime}\left(1+\zeta^{x b}\right) .
\end{aligned}
$$

Now $V^{\prime}\left(1+\zeta^{x b}\right)$ is odd if and only if $x b$ is odd since $1+\zeta^{x b}$ is a prime element of $K_{\pi}\left(\varepsilon_{4}\right)$ when $x b$ is odd. Thus from the definition of the Hasse invariant we get

$$
\begin{aligned}
\operatorname{inv}\left(K_{\pi} \otimes \Delta_{q}\right) & =0 & & \text { if } x b \text { is even } \\
& =1 / 2 & & \text { if } x b \text { is odd. }
\end{aligned}
$$

Thus

$$
\operatorname{inv}_{2}\left[\Delta_{q}\right]=1 / 2 \Longleftrightarrow \mu_{0} x b \quad \text { is odd }
$$

where

$$
\begin{array}{rlr}
\mu_{0}=0 & \text { if } 2 \text { is unramified in } L / K \\
=1 & \text { if } 2 \text { is ramified in } L / K
\end{array}
$$

Observe that $q$ and the primes which divide $n$ are the only primes which might ramify in $L\left(\varepsilon_{q}\right) / K$. Hence, these are the only primes at which $\Delta_{q}$ can have nonzero invariants.

THEOREM 3. The 2-primary part of $S(K)$ consists of all classes [A] in $B(K)$ with uniformly distributed invariants of value 0 or 1/2 which satisfy the following conditions. 
( I ) For a prime $p$ which divides $n$, $\operatorname{inv}_{p}[A]=0$ if any of the following hold:

( a ) $e(p)$ is odd;

(b) $f(p)$ is even;

(c) $[L: K(\zeta)] / e(p)$ is an even integer.

(II) For $q$ a prime which does not divide $n$, $\operatorname{inv}_{q}[A]=0$ if either

(a) $t=s-r$ and $f(q)$ is even, or

(b) $t \neq s-r, f(q)$ is even, and $q^{f(q)} \equiv(-h)^{g} \bmod 2^{s+1}$ where $[L / K, q]=\phi^{g}$.

(III) Let $p$ be a prime which divides $n$ such that condition (I) does not apply to $p$. If $|e(p, K / Q)|_{2} \geqq\left|e\left(p^{\prime}, K / Q\right)\right|_{2}$ for every prime $p^{\prime} \neq p$, then the invariant of $[A]$ is $1 / 2$ at an even number of primes in the set

$$
\{p\} \cup\{\text { primes } q:(p / q)=-1,(q, n)=1\}
$$

where $(p / q)$ is the Legendre symbol.

Proof. We have assumed that $\langle\phi\rangle$ has even order. Hence, by Lemma 2, we may assume that $[L: K]=2^{c}$.

First suppose that $K$ is a real field. Pick an odd prime of $q$ such that $f(q) e(q, K / Q)$ is even. There will always be such a prime since $[K: Q]$ must be even. Then the algebra $K \otimes_{Q}\left(Q\left(\varepsilon_{q}\right), \tau,-1\right)$ where $\langle\tau\rangle=\operatorname{Gal}\left(Q\left(\varepsilon_{q}\right) / Q\right)$ has invariant $1 / 2$ only at the infinite primes of $K$. Thus $\Omega\left(p_{\infty}\right)$ is in $S(K)_{2}$ when $K$ is real.

For the rest of the proof, "prime" will mean "finite prime."

Step 1. Condition (I) is satisfied.

Let $p$ be a prime which divides $n$. If $e(p)=1$, then $p$ is unramified in $L\left(\varepsilon_{q}\right) / K$ for any prime $q$ not dividing $n$. Hence $\operatorname{inv}_{p}[A]=0$ for all $[A]$ in $S(K)_{2}$. Now suppose that $e(p)$ is even.

If $p \neq 2$ and $\left\langle\phi^{a}\right\rangle$ is the intertia group of $p$ in $L / K$, then $2^{s-r}$ divides $a$, or if $s=r, 2$ divides $a$. Since the power of 2 dividing $a$ must divide $(p-1) / e(p)$, we have that $t(p) / e(p)$ is even. Further $h=5^{2^{r-2}}$ so $\left(h^{a}-1\right) /(h+1)$ is not divisible by $2^{s}$ if and only if $2^{s-r+1}$ does not divide $a$, or if $s=r$, if and only if 4 does not divide $a$. However this happens if and only if $[L: K]=2^{s-r} e(p)$, or if $s=r$, if and only if $[L: K]=2 e(p)$. Thus we have

$$
\frac{h^{a}-1}{h+1} \not \equiv 0 \bmod 2^{s} \Longleftrightarrow[L: K(\zeta)] / e(p) \quad \text { is odd . }
$$

Let $q$ be a prime which does not divide $n$ and let $\gamma^{b} \phi^{b \prime}$ be a 
Frobenius automorphism of $p$ in $L\left(\varepsilon_{q}\right) / K$ where $\langle\gamma\rangle=\operatorname{Gal}\left(L\left(\varepsilon_{q}\right) / L\right)$. Then we may rewrite (3.8) to read

$$
\operatorname{inv}_{p}\left[\Delta_{q}\right]=1 / 2 \Longleftrightarrow([L: K(\zeta)] / e(p)) x b \quad \text { is odd }
$$

since $2^{s-1}$ divides $z$. Since $b$ is even if $f(p)$ is even, (3.10) implies condition (I) for $p \neq 2$.

If $\gamma^{b}$ is a Frobenius automorphism for 2 in $L\left(\varepsilon_{q}\right) / K$, then $b$ is even if $f(2)$ is even. Thus (3.9) gives condition $(\mathrm{I})(\mathrm{b})$. Since $\operatorname{Gal}(L / K)=\langle\rho\rangle$ when 2 is ramified in $L / K$, we see that condition (I) (c) never applies to 2.

Step 2. Condition (II) holds.

Let $q$ be a prime not dividing $n$ and let $[L / K, q]=\phi^{g}$. We consider the invariants of algebras of the form $\Delta_{q}=\Delta_{q}(x, y, z)$. We have

$$
\phi^{g}(\zeta)=\zeta^{(-h)^{g}}=\zeta^{q^{f(q)}} .
$$

Hence $q^{f(q)}=(-h)^{g}+V 2^{s}$ for some integer $V$. Further, by (3.6) (b), we have

$$
y=\frac{x(q-1)+W 2^{s}}{1+h}
$$

for some integer $W$. Thus we may rewrite (3.7) to read

(3.11) $\operatorname{inv}_{q}\left[\Delta_{q}\right]=1 / 2 \Longleftrightarrow\left(\frac{W}{1+h}\right)\left(\frac{q^{f(q)}-1}{q-1}\right)+\frac{x V}{h+1} \equiv 1 / 2 \bmod Z$.

Now $t(q) /(q-1)$ is even if $f(q)$ is even. Moreover $x$ is even if $t=s-r$ and $V$ is even if $q^{f(q)} \equiv(-h)^{g} \bmod 2^{s+1}$. Hence condition (II) is obtained directly from (3.11).

Step 3. For each prime $l$ to which conditions (I) and (II) do not apply, there is a class $[A]$ in $S(K)_{2}$ such that $\operatorname{inv}_{l}[A]=1 / 2$.

Suppose that $q$ is a prime which does not divide $n$ such that condition (II) does not apply to $q$. If $f(q)$ is odd, then the algebra

$$
\Delta_{q}^{0}=\Delta_{q}\left(0,2^{s-1}, 0\right)
$$

has invariant $1 / 2$ at $q$ since $W=(h+1) / 2$ is odd.

If $f(q)$ is even, $t \neq s-r$, and $q^{f(q)} \not \equiv(-h)^{g} \bmod 2^{s+1}$, then consider the algebra

$$
\Delta_{q}^{\prime}=\Delta_{q}\left(\frac{h+1}{2}, \frac{q-1}{2}, 0\right) .
$$


We have that $t(q) /(q-1)$ is even and that $V$ is odd, thus (3.11) implies that $\operatorname{inv}_{q}\left[\Delta_{q}^{\prime}\right]=1 / 2$.

Now let $p$ be a prime which divides $n$ such that condition (I) does not apply to $p$. Pick a prime $q$ which does not divide $n$ such that $\left[Q\left(\varepsilon_{4 p}\right) / Q, q\right]=\psi_{p}$, where $\psi_{p}$ generates $\operatorname{Gal}\left(Q\left(\varepsilon_{4 p}\right) / Q\left(\varepsilon_{4}\right)\right)$. This choice of $q$ insures that $q \equiv 1 \bmod 4$ and that $(q / p)=-1$. Hence, by quadratic reciprocity, $(p / q)=-1$ so that $b$ is odd where $\gamma^{b} \phi^{b^{\prime}}$ is a Frobenius automorphism of $p$ in $L\left(\varepsilon_{q}\right) / K$ and $\langle\gamma\rangle=\operatorname{Gal}\left(L\left(\varepsilon_{q}\right) / L\right)$. Hence, by (3.10) and (3.9) $\operatorname{inv}_{p}\left[\Delta_{q}^{\prime}\right]=1 / 2$.

Step 4. If condition (III) does not apply, then $\Omega(l)$ is in $S(K)_{2}$ for every prime $l$ to which conditions (I) and (II) do not apply.

Let $p$ be a prime dividing $n$ to which condition (I) does not apply. Then $p$ is totally ramified in $L / K(\zeta)$. Further, since the inertia group of a prime in $Q\left(\varepsilon_{n}\right) / K$ must be a subgroup of its inertia group in $Q\left(\varepsilon_{n}\right) / Q$, we have that $p$ is the only prime which is ramified in $L / K$. Thus $p$ is the only prime dividing $n$ to which condition (I) does not apply.

Suppose that $|e(p, K / Q)|_{2}<\left|e\left(p^{\prime}, K / Q\right)\right|_{2}$ for some prime $p^{\prime} \neq p$ which divides $n$. Let $2^{\lambda}=|e(p, K / Q)|_{2}$.

(a) Assume that $p^{\prime}$ is odd.

Pick a prime $q_{0}$ not dividing $n$ such that $\left[L / Q, q_{0}\right]=\psi_{p} \psi_{p^{\prime}}$ where $\left\langle\psi_{p^{\prime}}\right\rangle=\operatorname{Gal}\left(Q\left(\varepsilon_{p^{\prime}}\right) / Q\right)$ and $\psi_{p}=\psi$ if $p=2$ or $\left\langle\psi_{p}\right\rangle=\operatorname{Gal}\left(Q\left(\varepsilon_{p}\right) / Q\right)$ if $p \neq 2$. There are infinitely many such $q_{0}$ by the Tchebotarev density theorem. Our choice of $q_{0}$ insures that $q_{0} \equiv 5 \bmod 8$ if $p=2$ or $\left(q_{0} / p\right)=-1$ if $p \neq 2$. Thus $\left(p / q_{0}\right)=-1$ since $q_{0} \equiv 1 \bmod 4$ by choice. Let $\gamma$ generate $\operatorname{Gal}\left(L\left(\varepsilon_{q_{0}}\right) / L\right)$ and let $\gamma^{b} \phi^{b^{\prime}}$ be a Frobenius automorphism for $p$ in $L\left(\varepsilon_{q_{0}}\right) / K$. Then $b$ must be odd. Thus inv $v_{p}\left[\Delta_{q_{0}}^{\prime}\right]=$ $1 / 2$ by (3.9) and (3.10). On the other hand, $f\left(q_{0}\right)$ is divisible by $\left|p^{\prime}-1\right|_{2}$ since $\left[L / K, q_{0}\right] \in \operatorname{Gal}(L / K(\zeta))$ if $p \neq 2$ and $\left[L / K, q_{0}\right]=1$ if $p=2$. Hence $q_{0}^{f\left(q_{0}\right)}$ and $h^{g}$, where $\left[L / K, q_{0}\right]=\phi^{g}$, are both equivalent to 1 modulo $2^{s+1}$. This is clear if $p=2$; if $p \neq 2$, then $q_{0} \equiv 1 \bmod 2^{s}$ and $\phi^{g}$ must be a square in Gal $(L / K(\zeta))$ by our choice of $q_{0}$. Thus condition (II) applies to $q_{0}$, so $\operatorname{inv}_{q_{0}}\left[\Delta_{q_{0}}\right]=0$. Hence $\Omega(p)=\left[\Delta_{q_{0}}^{\prime}\right]$.

(b) Assume that $p^{\prime}=2$, that is that $2^{s-2}>2^{\lambda}$.

Pick a prime $q_{1}$ not dividing $n$ such that $\left[L\left(\varepsilon_{2^{s+1}}\right) / Q, q^{\prime}\right]=$ $\psi_{p} \psi_{2^{\prime}}^{2 s-\lambda-2}$, where $\psi_{p}$ is the generator of the Sylow-2 subgroup of Gal $\left(Q\left(\varepsilon_{p}\right) / Q\right)$ such that $\psi_{p}^{2^{\lambda+r-s}}\left(\varepsilon_{p}\right)=\phi\left(\varepsilon_{p}\right)$, and $\psi_{2^{\prime}}$ is the automorphism sending $\varepsilon_{2^{s+1}}$ to $\varepsilon_{2^{8+1}}^{5}$. Now

$$
\left[L\left(\varepsilon_{2^{s+1}}\right) / K, q_{1}\right]=\left(\psi_{p}^{2 \lambda+r-s} \psi_{2^{\prime}}^{2 r-2}\right)^{g}
$$

for some $g, 2 \leqq g \leqq 2^{s-r}$. Hence $\left[L / K, q_{1}\right]=\phi^{g}$. Further, 


$$
\psi_{2^{\prime}}^{2 r-2 g}\left(\varepsilon_{2^{s+1}}\right)=\left(\varepsilon_{2^{s+1}}\right)^{h^{g}}=\left(\varepsilon_{2^{8+1}}\right)^{q_{1} f\left(q_{1}\right)},
$$

so $h^{g} \equiv q_{1}^{f\left(q_{1}\right)} \bmod 2^{s+1}$. This implies that $\operatorname{inv}_{q_{1}}\left[\Delta_{q_{1}}^{\prime}\right]=0$ since we arranged for $f\left(q_{1}\right)$ to be even.

On the other hand, we picked $q_{1}$ so that $q_{1} \equiv 1 \bmod 4$ and $\left(q_{1} / p\right)=-1$. Hence $\left(p / q_{1}\right)=-1$. Thus, by (3.10), inv ${ }_{p}\left[\Delta_{q_{1}}^{\prime}\right]=1 / 2$. Therefore $\Omega(p)=\left[\Delta_{q}^{\prime}\right]$.

Now let $q$ be a prime which does not divide $n$ such that condition (II) does not apply to $q$. By Step 3 , there is an algebra $\Delta_{q}^{*}$ such that $\operatorname{inv}_{q}\left[\Delta_{q}^{*}\right]=1 / 2$. If $\operatorname{inv}_{p}\left[\Delta_{q}^{*}\right]=0$, then $\Omega(q)=\left[\Delta_{q}^{*}\right]$. If $\operatorname{inv}_{p}\left[\Delta_{q}^{*}\right]=$ $1 / 2$, then $\Omega(q)=\left[\Delta_{q}^{*}\right] \otimes_{K} \Omega(p)$.

Step 5. Condition (III) holds.

Suppose that $p$ is a prime dividing $n$ to which condition (I) does not apply. Further suppose that $|e(p, K / Q)|_{2} \geqq\left|e\left(p^{\prime}, K / Q\right)\right|_{2}$ for every prime $p^{\prime} \neq p$ which divides $n$.

Let $q$ be a prime not dividing $n$. Let $\langle\gamma\rangle=\operatorname{Gal}\left(L\left(\varepsilon_{q}\right) / L\right)$ and $\gamma^{b} \phi^{b^{\prime}}$ be a Frobenius automorphism for $p$ in $L\left(\varepsilon_{q}\right) / K$.

First suppose that $\operatorname{inv}_{p}\left[\Delta_{q}^{*}\right]=1 / 2$ where $\Delta_{q}^{*}$ is an algebra of the form $\Delta_{q}$. From (3.9) and (3.10) we see that this implies that $x b$ is odd. Thus $b$ is odd, which means that $(p / q)=-1$. Further, if $p \neq 2$, then our hypotheses insure that $p \equiv 1 \bmod 4$. Thus $(q / p)=-1$ if $p \neq 2$, or $q \equiv 3$ or $5 \bmod 8$ if $p=2$. Suppose $p \neq 2$, then $\left|e(p, K / Q) / 2^{s-r}\right|_{2}>2^{r-2}$, so the full 2-part of $e(p, K / Q)$ is equal to $|f(q)|_{2}$. Hence $q^{f(q)} \equiv 1 \bmod 2^{s+1} \quad$ and $[L / K, q]=\phi^{2^{2-r}}$. Since $h^{2^{s-r}} \not \equiv 1 \bmod 2^{s+1}$, we have by (3.11) that $\operatorname{inv}_{q}\left[\Delta_{q}^{*}\right]=1 / 2$. In the case where $p=2, \quad|f(q)|_{2}=2^{s-2}$ so $q^{f(q)} \not \equiv 1 \bmod 2^{s+1}$. However $[L / K, q]=1$. Thus, by (3.11), $\operatorname{inv}_{q}\left[\Delta_{q}^{*}\right]=1 / 2$.

Now suppose that $(p / q)=-1$ and $\operatorname{inv}_{q}\left[\Delta_{q}^{*}\right]=1 / 2$. Since $(p / q)=-1$ we have that $b$ is odd. Further, $(q / p)=-1$ if $p \neq 2$ or $q \equiv 3$ or $5 \bmod 8$ if $p=2$. Hence $f(q)$ is divisible by $\left|e(p, K / Q) / 2^{s-r}\right|_{2}$ if $p \neq 2$ or by $2^{s-r}$ if $p=2$. This means that $f(q)$ is even so that $x v$ is odd. Thus $x b$ is odd. Hence (3.9) and (3.10) imply that $\operatorname{inv}_{p}\left[\Delta_{q}^{*}\right]=1 / 2$.

We have just shown that

$$
\operatorname{inv}_{p}\left[\Delta_{q}\right]=1 / 2 \Longleftrightarrow \operatorname{inv}_{q}\left[\Delta_{q}\right]=1 / 2 \text { and }(q / p)=-1 .
$$

Since every algebra class $[A]$ in $S(K)_{2}$ is a product of classes of the form $\left[\Delta_{q}\right]$, this gives condition (III).

In addition, this shows that $\Omega(q)$ is in $S(K)_{2}$ where $q$ is a prime not dividing $n$ such that $(q / p)=1$ and condition (II) does not apply to $q$. This is because there is an algebra $\left[\Delta_{q}^{*}\right]$ with $\operatorname{inv}_{q}\left[\Delta_{q}^{*}\right]=1 / 2$ by Step 3 , and we have just seen that $\operatorname{inv}_{p}\left[\Delta_{q}^{*}\right]=0$. 
This completes the proof of the theorem.

We have now determined the Schur subgroup of all fields $K$, not containing a fourth root of unity, which have a cyclic extension of the form $Q\left(\varepsilon_{n}\right)$. Observe that subfields of $Q\left(\varepsilon_{p} d\right)$ are included as special cases. The Schur group of these fields was first found by Yamada [8].

\section{REFERENCES}

1. A. A. Albert, Structure of Algebras, Amer. Math. Soc. Colloq. Publications XXIV, Providence, 1961.

2. M. Bernard, The Schur subgroup, I, J. of Algebra, 22, No. 2 (1972), 374-377.

3. M. Bernard and M. Schacher, The Schur subgroup, II, J. of Algebra, 22, No. 2 (1972), 378-385.

4. K. L. Fields, On the Brauer-Speiser theorem, Bull. Amer. Math. Soc., 77, No. 2 (1971), 477-478.

5. G. J. Janusz, The Schur group of cyclotomic field, J. of Number Theory, (to appear). 6. - Generators for the Schur group of local and global number fields, Pacific J. Math., (to appear).

7. T. Yamada, Characterization of the simple components of the group algebra over the p-adic number field, J. Math. Soc. Japan, 23 (1971), 295-310.

8. The Schur subgroup of the Brauer group, I, J. of Algebra, 27 (1973), $579-589$.

Received November 18, 1974. The material presented in this paper is from the author's doctoral thesis, written at the University of Illinois under the direction of Professor G. J. Janusz.

New Mexico State University

Las Cruces, NM 88001 



\section{PACIFIC JOURNAL OF MATHEMATICS}

\section{EDITORS}

RICHARD ARENS (Managing Editor)

University of California

Los Angeles, CA 90024

R. A. BEAUMONT

University of Washington

Seattle, WA 98105

C. C. Moore

University of California

Berkeley, CA 94720

\section{J. DugundJI}

Department of Mathematics

University of Southern California

Los Angeles, CA 90007

R. Finn and J. Milgram

Stanford University

Stanford, CA 94305

\section{ASSOCIATE EDITORS}

\section{E. F. BECKENBACH}

B. H. NEUMANN

F. WoLF

K. YOSHIDA

\section{SUPPORTING INSTITUTIONS}

\author{
UNIVERSITY OF BRITISH COLUMBIA \\ CALIFORNIA INSTITUTE OF TECHNOLOGY \\ UNIVERSITY OF CALIFORNIA \\ MONTANA STATE UNIVERSITY \\ UNIVERSITY OF NEVADA \\ NEW MEXICO STATE UNIVERSITY \\ OREGON STATE UNIVERSITY \\ UNIVERSITY OF OREGON \\ OSAKA UNIVERSITY
}

\author{
UNIVERSITY OF SOUTHERN CALIFORNIA \\ STANFORD UNIVERSITY \\ UNIVERSITY OF HAWAII \\ UNIVERSITY OF TOKYO \\ UNIVERSITY OF UTAH \\ WASHINGTON STATE UNIVERSITY \\ UNIVERSITY OF WASHINGTON \\ AMERICAN MATHEMATICAL SOCIETY
}

The Supporting Institutions listed above contribute to the cost of publication of this Journal, but they are not owners or publishers and have no responsibility for its content or policies.

Mathematical papers intended for publication in the Pacific Jaurnal of Mathematics should be in typed form or offset-reproduced, (not dittoed), double spaced with large margins. Please do not use built up fractions in the text of your manuscript. You may however, use them in the displayed equations. Underline Greek letters in red, German in green, and script in blue. The first paragraph or two must be capable of being used separately as a synopsis of the entire paper. Items of the bibliography should not be cited there unless absolutely necessary, in which case they must be identified by author and Journal, rather than by item number. Manuscripts, in triplicate, may be sent to any one of the editors. Please classify according to the scheme of Math. Reviews, Index to Vol. 39. All other communications should be addressed to the managing editor, or Elaine Barth, University of California, Los Angeles, California, 90024.

The Pacific Journal of Mathematics expects the author's institution to pay page charges, and reserves the right to delay publication for nonpayment of charges in case of financial emergency

100 reprints are provided free for each article, only if page charges have been substantially paid. Additional copies may be obtained at cost in multiples of 50 .

The Pacific Journal of Mathematics is issued monthly as of January 1966. Regular subscription rate: $\$ 7200$ a year (6 Vols., 12 issues). Special rate: $\$ 36.00$ a year to individual members of supporting institutions.

Subscriptions, orders for back numbers, and changes of address should be sent to Pacific Journal of Mathematics, 103 Highland Boulevard, Berkeley, California, 94708.

PUBLISHED BY PACIFIC JOURNAL OF MATHEMATICS, A NON-PROFIT CORPORATION

Printed at Kokusai Bunken Insatsusha (International Academic Printing Co., Ltd.). 8-8, 3-chome, Takadanobaba, Shinjuku-ku, Tokyo 160, Japan.

Copyrit (C) 1975 by Pacific Journal of Mathematics Manufactured and first issued in Japan 


\section{Pacific Journal of Mathematics \\ Vol. 69, No. $2 \quad$ June, 1977}

Carol Alf and Thomas Alfonso O'Connor, Unimodality of the Lévy spectral

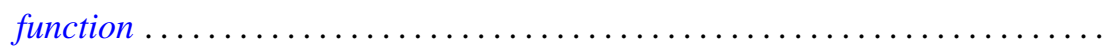

S. J. Bernau and Howard E. Lacey, Bicontractive projections and reordering of

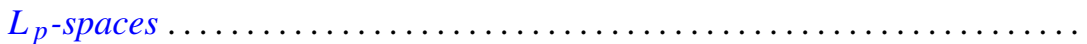

Andrew J. Berner, Products of compact spaces with bi-k and related spaces..... 303

Stephen Richard Bernfeld, The extendability and uniqueness of solutions of ordinary differential equations ...............................

Marilyn Breen, Decompositions for nonclosed planar m-convex sets ..........

Robert F. Brown, Cohomology of homomorphisms of Lie algebras and Lie

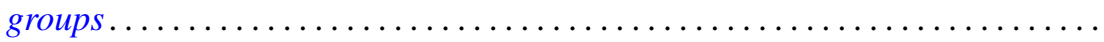

Jack Douglas Bryant and Thomas Francis McCabe, A note on Edelstein's iterative test and spaces of continuous functions ....................

Victor P. Camillo, Modules whose quotients have finite Goldie dimension ....... 333

David Downing and William A. Kirk, A generalization of Caristi's theorem with

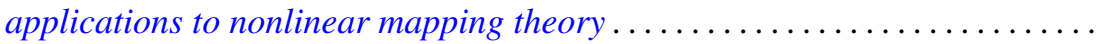

Daniel Reuven Farkas and Robert L. Snider, Noetherian fixed rings ...........

Alessandro Figà-Talamanca, Positive definite functions which vanish at

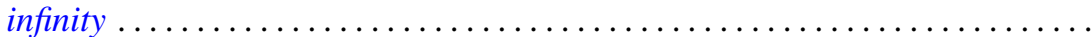

Josip Globevnik, The range of analytic extensions .................. 365

André Goldman, Mesures cylindriques, mesures vectorielles et questions de

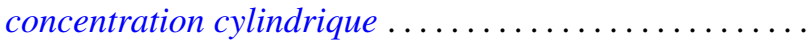

Richard Grassl, Multisectioned partitions of integers..........

Haruo Kitahara and Shinsuke Yorozu, A formula for the normal part of the

Laplace-Beltrami operator on the foliated manifold .... .

Marvin J. Kohn, Summability $R_{r}$ for double series .........

Charles Philip Lanski, Lie ideals and derivations in rings with involution ..

Solomon Leader, A topological characterization of Banach contractions . .

Daniel Francis Xavier O’Reilly, Cobordism classes of fiber bundles . .

James William Pendergrass, The Schur subgroup of the Brauer group . .

Howard Lewis Penn, Inner-outer factorization of functions whose Fourier series

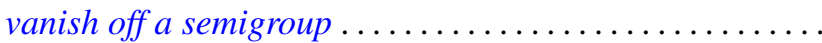

501

William T. Reid, Some results on the Floquet theory for disconjugate definite

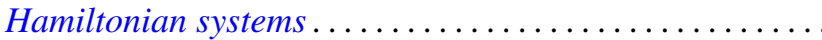

Caroll Vernon Riecke, Complementation in the lattice of convergence

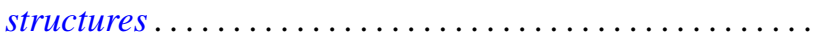

Louis Halle Rowen, Classes of rings torsion-free over their centers ......... 527

Manda Butchi Suryanarayana, A Sobolev space and a Darboux problem ....... 535

Charles Thomas Tucker, II, Riesz homomorphisms and positive linear maps.... 551

William W. Williams, Semigroups with identity on Peano continua ........... 557

Yukinobu Yajima, On spaces which have a closure-preserving cover by finite 